\title{
A urinary microRNA signature can predict the presence of bladder urothelial carcinoma in patients undergoing surveillance
}

\author{
Nikhil Sapre ${ }^{1}$, Geoff Macintyre ${ }^{2,3,4}$, Michael Clarkson ${ }^{1}$, Haroon Naeem ${ }^{2,3,4}$, Marek Cmero ${ }^{2}$, \\ Adam Kowalczyk ${ }^{2,3,4}$, Paul D Anderson ${ }^{1}$, Anthony J Costello ${ }^{1}$, Niall M Corcoran ${ }^{1}$ and Christopher M Hovens ${ }^{*, 1}$ \\ ${ }^{1}$ Department of Surgery, Division of Urology, Royal Melbourne Hospital, The University of Melbourne, Parkville, Melbourne, \\ Victoria, Australia; ${ }^{2}$ NICTA Victoria Research Laboratory, Department of Electronic Engineering, University of Melbourne, \\ Melbourne, Victoria, Australia; ${ }^{3}$ Department of Computing and Information Systems, University of Melbourne, Melbourne, \\ Victoria, Australia and ${ }^{4}$ Centre for Neural Engineering, University of Melbourne, Melbourne, Victoria, Australia
}

Background: The objective of this study was to determine whether microRNA (miRNA) profiling of urine could identify the presence of urothelial carcinoma of the bladder (UCB) and to compare its performance characteristics to that of cystoscopy.

\begin{abstract}
Methods: In the discovery cohort we screened 81 patients, which included 21 benign controls, 30 non-recurrers and 30 patients with active cancer (recurrers), using a panel of 12 miRNAs. Data analysis was performed using a machine learning approach of a Support Vector Machine classifier with a Student's t-test feature selection procedure. This was trained using a three-fold cross validation approach and performance was measured using the area under the receiver operator characteristic curve (AUC). The miRNA signature was validated in an independent cohort of a further 50 patients.
\end{abstract}

Results: The best predictor to distinguish patients with UCB from non-recurrers was achieved using a combination of six miRNAs (AUC $=0.85)$. This validated in an independent cohort $(A \cup C=0.74)$ and detected UCB with a high sensitivity $(88 \%)$ and sufficient specificity (48\%) with all significant cancers identified. The performance of the classifier was best in detecting clinically significant disease such as presence of $T 1$ Stage disease $(A \cup C=0.92)$ and high-volume disease $(A \cup C=0.81)$. Cystoscopy rates in the validation cohort would have been reduced by $30 \%$.

Conclusions: Urinary profiling using this panel of miRNAs shows promise for detection of tumour recurrence in the surveillance of UCB. Such a panel may be useful in reducing the morbidity and costs associated with cystoscopic surveillance, and now merits prospective evaluation.

Up to $15 \%$ of patients presenting with macroscopic haematuria are diagnosed with urothelial carcinoma of the bladder (UCB) with the figure for microscopic haematuria being 4.8\% (Khadra et al, 2000). UCB has a high propensity of recurrence and progression, which necessitates extensive surveillance (Sylvester et al, 2006). There are no non-invasive markers to predict recurrence, which currently relies on invasive procedures such as cystoscopy. This has made the treatment of UCB resource intensive and it is now the single most expensive cancer to treat from diagnosis to death (Botteman et al, 2003; Avritscher et al, 2006). Furthermore, more than half of these costs are towards treating the non-muscle invasive spectrum of the disease (Sangar et al, 2005). The morbidity of cystoscopy is often under-estimated and patient adherence with surveillance has been reported to be as low as $40 \%$ (Schrag et al, 2003). In addition, flexible cystoscopy has a definite false negative rate (Daniltchenko et al, 2005).

There is a pressing need for an accurate non-invasive test to assist diagnosis and surveillance of UCB. Urine is in direct contact with bladder cancer cells, and hence is an ideal source for investigation of non-invasive biomarkers of UCB. While many

*Correspondence: Professor CM Hovens; E-mail: chovens@unimelb.edu.au

Received 11 August 2015; revised 28 November 2015; accepted 3 December 2015; published online 26 January 2016

(c) 2016 Cancer Research UK. All rights reserved 0007-0920/16 
urinary markers have been studied, few, if any, of these markers have been validated in independent cohorts (Sapre et al, 2014). Even fewer have been deemed robust enough for incorporation into clinical practice.

A class of biomarkers that has recently been investigated is microRNAs. MicroRNAs (miRNAs) are $\sim 22$-nucleotide long, single-stranded, non-coding RNAs that bind to complementary 'seed' regions found in $3^{\prime}$-untranslated region (UTR) of particular messenger RNA (mRNA) species. MiRNAs modulate expression of their mRNA targets, either marking them for destruction or inhibiting their binding to translational machinery. MiRNAs have been shown to be involved in a wide range of important physiological and pathological processes including cell cycle processes, development, survival, differentiation, growth, apoptosis and immune response (Jansson and Lund, 2012).

There are several advantages of miRNAs as biomarkers in biofluids. Biofluids such as plasma and urine are less invasive to obtain compared with tissue, and miRNAs are frequently deregulated in cancer and exhibit tissue-specific expression (Garzon et al, 2009). In addition, their expression in blood and urine is stable and can be quantified sensitively by quantitative real-time polymerase chain reaction (qRT-PCR) (Hanke et al, 2010). Many have studied the regulation of miRNA species in bladder cancer tissues (Catto et al, 2011). In addition, many of the miRNAs involved in bladder cancer initiation, progression and metastasis have been functionally characterised (Burk et al, 2008, Adam et al, 2009, Mongroo and Rustgi, 2010). Recent work also profiles miRNA species found to be up- or downregulated in urine of bladder cancer patients (Hanke et al, 2010; Miah et al, 2012; Wang et al, 2012; Yun et al, 2012). However, none of these studies has investigated the use of these biomarkers in the surveillance setting where patients who have all been previously diagnosed with UCB are screened and few of these studies have performed independent validation of the defined biomarkers. These limitations typically preclude the clinical translation of biomarker studies in the management of bladder cancer.

We wanted to systematically investigate the role of miRNAs as urinary biomarkers for detection of UCB. The aim of this study was to investigate if the profiling of a panel of miRNAs in the urine of patients can distinguish the patients with cancer in their bladder in the surveillance setting and to validate any significant findings in an independent cohort of patients.

\section{MATERIALS AND METHODS}

Patient selection. In total, 131 patients were analysed in this study comprising discovery and validation cohorts. Patients were selected for the initial discovery cohort from three distinct groups: (1) Patients without any previous history of UCB $(n=21)$. (2) Patients with a previous history of UCB but exhibiting no recurrence at cystoscopy (cancer-absent group, $n=30$ ) and (3) patients with active UCB at cystoscopy at initial diagnosis or during surveillance (cancer-present group, $n=30$ ) for a total of 81 patients. In the independent validation cohort we screened urine samples from active bladder cancer surveillance patients $(n=50)$, which included patients from a cancer-present group $(n=25)$ and patients from a cancerabsent group $(n=25)$. Tumours were classified according to the TNM and WHO/ISUP staging and grading (Epstein et al, 1998). Tumours larger than $3 \mathrm{~cm}$ were termed large or high-volume tumours.

MicroRNA panel selection. A systematic literature review was conducted in PubMed to find microRNAs implicated in epithelial cancer initiation, progression and metastasis using the terms 'cancer', 'bladder cancer' and 'microRNA' in January 2012. The cited references of studies were checked for further articles of interest. All articles including original articles, reviews and abstracts were considered. Based on our review we chose a panel of 12 microRNAs all of which had at least two independent studies published showing involvement in epithelial cancer carcinogenesis, with preference given to those with concomitant supporting mechanistic data (Supplementary Table S1).

Nucleic acid extraction. Freshly voided urine was kept on ice and stored in a biorepository at $-180^{\circ} \mathrm{C}$ within $4 \mathrm{~h}$. A volume of $500 \mu \mathrm{l}$ of urine was thawed on ice for total RNA extraction using the mirVana miRNA Isolation Kit (Ambion, TX, USA) according to the following protocol. Volumes of reagents were as follows: urine: $500 \mu \mathrm{l}$, lysis buffer: $750 \mu \mathrm{l}, 10 \%$ homogenate: $125 \mu \mathrm{l}$, phenol chloroform: $1250 \mu \mathrm{l}$. On ice, $1.5 \times$ volume of lysis buffer olution was added to the urine sample and vortexed for $30 \mathrm{~s}$. On ice, $1 / 10$ volume of miRNA homogenate additive was added, vortexed for $10 \mathrm{~s}$ and left on ice for $10 \mathrm{~min}$. In the fume hood, an identical volume of acid-phenol:chloroform as the initial lysate (urine input + lysis buffer) was added and vortexed for $30 \mathrm{~s}$. The mixture was centrifuged for $5 \mathrm{~min}$ at $10000 \mathrm{~g}$, and the upper aqueous phase transferred to a new tube noting the volume. $1.25 \times$ volume of $100 \%$ ethanol was added to the aqueous phase. Aliquots of $700 \mu \mathrm{l}$ of this solution were placed on a filter cartridge/collection tube, and centrifuged at $10000 \mathrm{~g}$ for $15 \mathrm{~s}$. The flow-through was discarded and the procedure repeated until all the sample had gone through. $700 \mu \mathrm{l}$ miRNA wash solution 1 was added to the collection tube and centrifuged for $10 \mathrm{~s}$. The flow-through was discarded. A volume of $500 \mu \mathrm{l}$ wash solution $2 / 3$ was added to the collection tube and centrifuged for $10 \mathrm{~s}$. Flow-through was discarded. A second wash was performed with this solution. The collection tube was subjected to a 1-min dry spin. The filter cartridge was transferred to a labelled tube. A volume of $40 \mu \mathrm{l}$ pre-heated water was added to the filter and centrifuged for $1 \mathrm{~min}$. RNA elutions were frozen on dry ice and stored in $-80^{\circ} \mathrm{C}$. RNA was concentrated using a Savant SpeedVac (Thermo Fisher Scientific, NC, USA) at $45^{\circ} \mathrm{C}$ at high pressure and reconstituted in $15 \mu$ l water.

cDNA library preparation and preamplification. Reverse transcription was performed on a thermal cycler (Applied Biosystems, CA, USA) using the Taqman microRNA Reverse Transcription Kit using a modification of the manufacturer's small RNA assay protocol using $7 \mu \mathrm{l}$ concentrated RNA, $100 \mathrm{~mm}$ deoxynucletide triphosphates, $50 \mathrm{U} \mu \mathrm{l}^{-1}$ Multiscribe Reverse Transcriptase, $10 \times$ reverse transcription buffer, $20 \mathrm{U}^{-1}$ RNAse inhibitor and nuclease-free water. Validated primers were pooled according to the manufacturer's instructions (Taqman, Applied Biosystems) using $10 \mu \mathrm{l} 5 \times$ primer for each primer to a total volume of $1 \mathrm{ml}$ using nuclease-free water. This reaction was subjected to the following conditions: $30 \mathrm{~min}$ at $16^{\circ} \mathrm{C}, 30 \mathrm{~min}$ at $42^{\circ} \mathrm{C}$ and $5 \mathrm{~min}$ at $85^{\circ} \mathrm{C}$. cDNA was stored at $-20^{\circ} \mathrm{C}$. Preamplification of the synthesised cDNA was performed according to the manufacturer's instructions. Briefly $2.5 \mu \mathrm{l} \mathrm{cDNA}$ was added to $12.5 \mu \mathrm{l}$ preamplification MasterMix, $3.75 \mu \mathrm{l}$ primer pool and $6.25 \mu \mathrm{l}$ nuclease-free water. This reaction was heated to $95^{\circ} \mathrm{C}$ for $10 \mathrm{~min}, 55^{\circ} \mathrm{C}$ for $2 \mathrm{~min}$ and $72{ }^{\circ} \mathrm{C}$ for $2 \mathrm{~min}$ followed by 12 cycles of amplification before heating to $99^{\circ} \mathrm{C}$ for $10 \mathrm{~min}$. The amplified reaction products were diluted to $200 \mu \mathrm{l}$ using $0.1 \times$ Tris-EDTA buffer $(\mathrm{pH}=8)$.

Polymerase chain reaction and microRNA detection. RT-qPCR was perfomed on the ViiA 7 (Applied Biosystems) using $0.5 \mu \mathrm{l}$ $20 \times$ miRNA assay, $0.1 \mu \mathrm{l}$ preamp product, $5.0 \mu \mathrm{l}$ TaqMan Fast Universal PCR Master Mix $(2 \times), 4.4 \mu \mathrm{l}$ nuclease-free water were combined for a $10-\mu$ l PCR reaction. PCR was conducted under the following conditions: $50{ }^{\circ} \mathrm{C}$ for $2 \mathrm{~min}$ followed by 40 cycles of amplification. Thresholds for the PCR runs were set using RQ Manager (Applied Biosystems) and checked manually to ensure the $\mathrm{Ct}$ corresponded to the midpoint of the logarithmic amplification. All reactions were performed in triplicate and the median included in the final analysis. 
Histopathological confirmation. The core of bladder tissue was OCT embedded and sectioned at $-24{ }^{\circ} \mathrm{C}$ in a cryostat (Leica Microsystems). A $5-\mu \mathrm{m}$ section was cut on to a slide and then deparaffinised with xylene then washed with a decreasing ethanol series (100, 95 and 70\%) and water. Slides were haematoxylin and eosin stained and again immersed in an increasing ethanol series (70, 95 and 100\%). Tumour status confirmation was obtained by an uropathologist prior for use in downstream experiments.

Tumour tissue processing and nucleic acid extraction. DNA and RNA were simultaneously extracted from tumour specimens using the Allprep Microkit (Qiagen, Melbourne, VIC, Australia). The frozen tissue core was placed in a tube containing $700 \mu \mathrm{l}$ of Buffer RLT Plus and $10 \mu \mathrm{ml}^{-1}$ mercaptoethanol. The TissueRuptor (Qiagen) was used to produce a homogenous lysate in three $10 \mathrm{~s}$ bursts. The homogenate was allowed to stand on ice for $15 \mathrm{~min}$ until foam settled and then transfer to a microcentrifuge tube. The lysate was centrifuged for $3 \mathrm{~min}$ at full speed and the supernatant carefully removed by pipetting and transferring it to an Allprep DNA spin column placed in a $2-\mathrm{ml}$ collection tube. This was centrifuged for $30 \mathrm{~s}$ at 10000 r.p.m. The Allprep spin column was placed in a new $2 \mathrm{ml}$ collection tube and stored at $4{ }^{\circ} \mathrm{C}$ for later DNA purification. The flow-through was used for RNA purification. The flow-through was added to $700 \mu \mathrm{l}$ of $70 \%$ ethanol and mixed well. The sample was then transferred to an RNEasy MinElute spin column placed in a $2-\mathrm{ml}$ collection tube and centrifuged for $15 \mathrm{~s}$ at 10000 r.p.m. and the flow-through discarded. A volume of $700 \mu \mathrm{l}$ Buffer RW1 was added to the RNEasy MinElute spin column and centrifuged for $15 \mathrm{~s}$ at 10000 r.p.m. and flow-through discarded. After DNase digestion on column for $15 \mathrm{~min}$, this step was repeated with $500 \mu \mathrm{l}$ of buffer RPE and $500 \mu \mathrm{l}$ of $80 \%$ ethanol to RNEasy MinElute spin column and centrifuged for $2 \mathrm{~min}$ at 10000 r.p.m. to wash the spin column membrane. This was followed by a 5-min dry spin. A volume of $15 \mu \mathrm{l}$ of RNase-free water was added to the centre of the spin column and centrifuged for $1 \mathrm{~min}$ at full speed to elute the RNA. This step was repeated again to obtain a final elution volume of $30 \mu \mathrm{l}$. RNA was stored at $-80^{\circ} \mathrm{C}$.

\section{Data analysis}

MicroRNA pre-processing. Thresholds for the PCR runs were set using RQ Manager (Applied Biosystems) and manually checked to ensure the $\mathrm{Ct}$ corresponded to the midpoint of the logarithmic amplification. All observed Ct values greater than 35 were considered not expressed and set to 35. Any undetermined Ct values were also set to 35 . As each miR was profiled in triplicate, any replicate value more than $20 \%$ different from the remaining two values was considered an outlier and removed from analysis. The mean $\mathrm{Ct}$ value was then determined for each sample and $\mathrm{miR}$ across replicates.

Normalisation. No endogenous control was used for normalisation in this study. Instead, urine concentrations as measured by urine osmolality $d_{\text {osm }}$ were used to normalise the Ct values of each miR so that they were comparable across patients by taking into account natural variation in urinary volumes and solute concentrations. To that end we set baseline osmolality $d_{0}=532.17$ ( $=$ the mean osmolality in the discovery cohort), then for each sample we added the correction $-\log _{2}\left(d_{\mathrm{osm}} / d_{0}\right)$ to each miR measured for that sample. This means that if $d_{\mathrm{osm}}>d_{0}$ for a particular sample, then each $\mathrm{Ct}$ value for this sample was decreased by the equivalent of $\log _{2}\left(d_{\mathrm{osm}} / d_{0}\right)$ cycles; if opposite, i.e., $d_{\mathrm{osm}}<d_{0}$, then the Ct for each miR was increased by $\log _{2}\left(d_{0} / d_{\mathrm{osm}}\right)$.

Differential expression analysis. The mean Ct of samples belonging to the cancer-present group or cancer-absent group was used to calculate fold-change for each $\mathrm{miR}$ as $2^{\text {(mean(Ct_present) - mean(Ct_absent) })}$. For fold-changes less than 1 , the negative inverse was taken and the fold change reported as negative (i.e., expressed less in the cancer-present group compared with cancer-absent group). A Student's $t$-test was used to calculate the significance of the difference of each miR's expression between cancer present and absent groups and the $P$-value was adjusted for multiple testing correction using the Benjamini-Hochberg method (Benjamini, 1995).

Feature selection and classification. We assume the data is given as a matrix $\left[x_{i j}\right], N$ features (miRs) for $M$ samples i.e., $1 \leqslant i \leqslant n$ and $1 \leqslant j \leqslant m$ with the label vector $\left[y_{i}\right]= \pm 1$ arranged in such a way that $\left[y_{j}\right]= \pm 1$ for $1 \leqslant j \leqslant m_{+}\left[y_{i}\right]=-1$ for the remaining samples $m_{+}<j \leqslant m$. We have used the Student's $t$-test for selection/ ordering of features/miRs from most to the least 'discriminating'. The Student's $t$-test, which allocated to the $i$-th feature the score

$$
t_{i}=\frac{\bar{x}_{+i}-\bar{x}_{-i}}{\sqrt{s_{+i}^{2}+s_{-i}^{2}}}
$$

where

$$
\bar{x}_{+i}=\frac{1}{m_{+}} \sum_{k=1}^{m_{+}} x_{i j_{k}} \quad \text { and } \quad \bar{x}_{-i}=\frac{1}{m_{-}} \sum_{k=m_{+}+1}^{m} x_{i j_{k}}
$$

denote the means and

$$
\begin{gathered}
s_{+i}^{2}=\frac{1}{m_{+}-1} \sum_{k=1}^{m_{+}}\left(x_{i j_{k}}-\bar{x}_{+i}\right)^{2} \text { and } \\
s_{-i}^{2}=\frac{1}{m_{-}-1} \sum_{k=m_{+}+1}^{m}\left(x_{i j_{k}}-\bar{x}_{-i}\right)^{2}
\end{gathered}
$$

denote the variances of the $i$-th variable for samples of the both groups of interest, respectively. Then the features are ranked in the descending order of absolute values of the statistic $\left|t_{i}\right|$, for $i=1,2, \ldots, n$.

For various subsets of $t$ top features $\left(x_{i_{1}}, x_{i_{2}}, \ldots, x_{i_{t}}\right)$, we generated linear classifiers

$$
f^{(t)}(x)=\sum_{k=1}^{t} w_{k} x_{i_{k}}+b,
$$

for a sample $\boldsymbol{x}=\left(x_{1}, \ldots, x_{n}\right)$, using a support vector machine algorithm, which selected weights $w_{k}$ and the intercept $b$ by minimising the following functional:

$$
\left(w_{k}, b\right)=\arg \min _{\left(w_{k}, b\right)} \sum_{k=1}^{t} w_{k}^{2}+\frac{C}{m} \sum_{j=1}^{m}\left(y_{j}-\sum_{k=1}^{t} w_{k} x_{i_{k}, j}-b\right)^{2} .
$$

Here $C$ is adjustable regularisation constant, with a relatively weak impact on the final result in our case. The results reported have used $C=1$.

The classifier trained was then applied systematically to the validation group samples and samples labelled as 'positive' or 'negative'. Sensitivity and specificity of the test were calculated by comparing the results with gold standard cystoscopy.

Adjusting for batch effects. Standard batch correction procedures such as Combat attempt to adjust the measurements of genes across different batches before any further analysis is completed (Kupfer et al, 2012). This approach was applicable in our setting when attempting to apply the classifier trained on the miRNA discovery cohort, to the validation cohort. However, an equally viable approach in this setting is to simply recalibrate the threshold of the score output by the classifier to achieve the best sensitivity and specificity on the validation cohort. We applied both approaches (data not shown) and both yielded similar performance. The results presented in this manuscript rely on 
recalibration of the classifier score, however, in a clinical setting, where the outcome of a sample is not known, standard batch correction methods should be used.

RNA-seq analysis. Samples were sequenced with $100 \mathrm{bp}$ singleend reads using Illumina HiSeq 2000. Raw sequencing data from different lanes were merged per sample. The sequence data was clipped for Illumina adapter sequences using Trimmomatic (Lohse et al, 2012). Reads were aligned to the 1000 genomes hg19 build 37 reference annotation using Tophat (Kim et al, 2013) v2.0.4 with default parameters and Bowtie (Langmead et al, 2009) v0.12.8.

Gene-level read counts were performed using genomeCoverageBed from Bedtools (Quinlan and Hall, 2010) v2.16.2 using RefSeq gene annotations. Differential expression was performed using the R Bioconductor package edgeR (Robinson et al, 2010). The significance threshold used was FDR (Benjamini-Hochberg adjusted $P$-value) of 0.05 .

Prediction of miR targets. miRNA target genes were identified using a suite of five prediction algorithms (miRanda, miRDB, miRWalk, RNA22 and TargetScan) included as the default search set in miRWalk (http://www.umm.uni-heidelberg.de/apps/zmf/ mirwalk/). For each miRNA, potential target genes were selected on the basis of being identified by three or more prediction algorithms and downregulated more than two-fold in our UCB expression analysis. As it is becoming apparent that a particular gene can be regulated by the coordinated action of a number of miRNAs (Krek et al, 2005), we decided to compile a list of genes that were also targeted by at least three of our six miRNAs.

\section{RESULTS}

To determine the viability of our selected panel of miRNAs as biomarkers for detecting UCB recurrence, we profiled a panel of 12
miRNAs using RT-qPCR in 81 patient samples (clinical characteristics summarised in Supplementary Table S2). These included patients with active $\operatorname{UCB}(n=30$; cancer-present group) and patients with a history of UCB but no recurrence at cystoscopy ( $n=30$; cancer-absent group). A benign control group $(n=21)$ was used as a reference panel to establish baseline levels of miRNAs present in benign urine. Figure 1 shows that all miRNAs except for miR129 were present at detectable levels in at least one sample from the cancer cohorts. All but one sample showed at least one miRNA at detectable levels in the urine. Unsupervised hierarchical clustering in Figure 1 also demonstrates a reasonable separation of patients with active UCB from non-recurrers. To explore this further, we used a comparison of the expression of each of the miRNAs between those patients with active UCB (tumour present) and those with a history of UC but no recurrence (tumour absent) as a measure of suitability of these miRNAs to detect the presence of a tumour in urine during follow-up. Differential expression analysis of the miRNAs (Supplementary Table S3), between the tumour present and tumour absent groups showed that 8 of the 12 miRNAs showed significantly higher expression in the tumour present group than the absent group suggesting that these miRNAs may be useful as biomarkers to distinguish the two groups.

An accurate and robust clinical test for detecting the presence of a tumour may not require measurement of all of these miRNAs, but rather a subset given that related miRNAs in the set may provide redundant information. Therefore, to determine the minimum number of miRNAs for accurate prediction of the presence of a tumour, we carried out a procedure whereby we built and tested the performance of different classifiers using increasing numbers of miRNAs. This analysis showed that performance in terms of AUC and accuracy did not increase significantly beyond the use of six miRNAs (Figure 2A). Therefore, we opted to build our biomarker test using these six miRNAs. To find the precise set of miRNAs that provided the best performance when

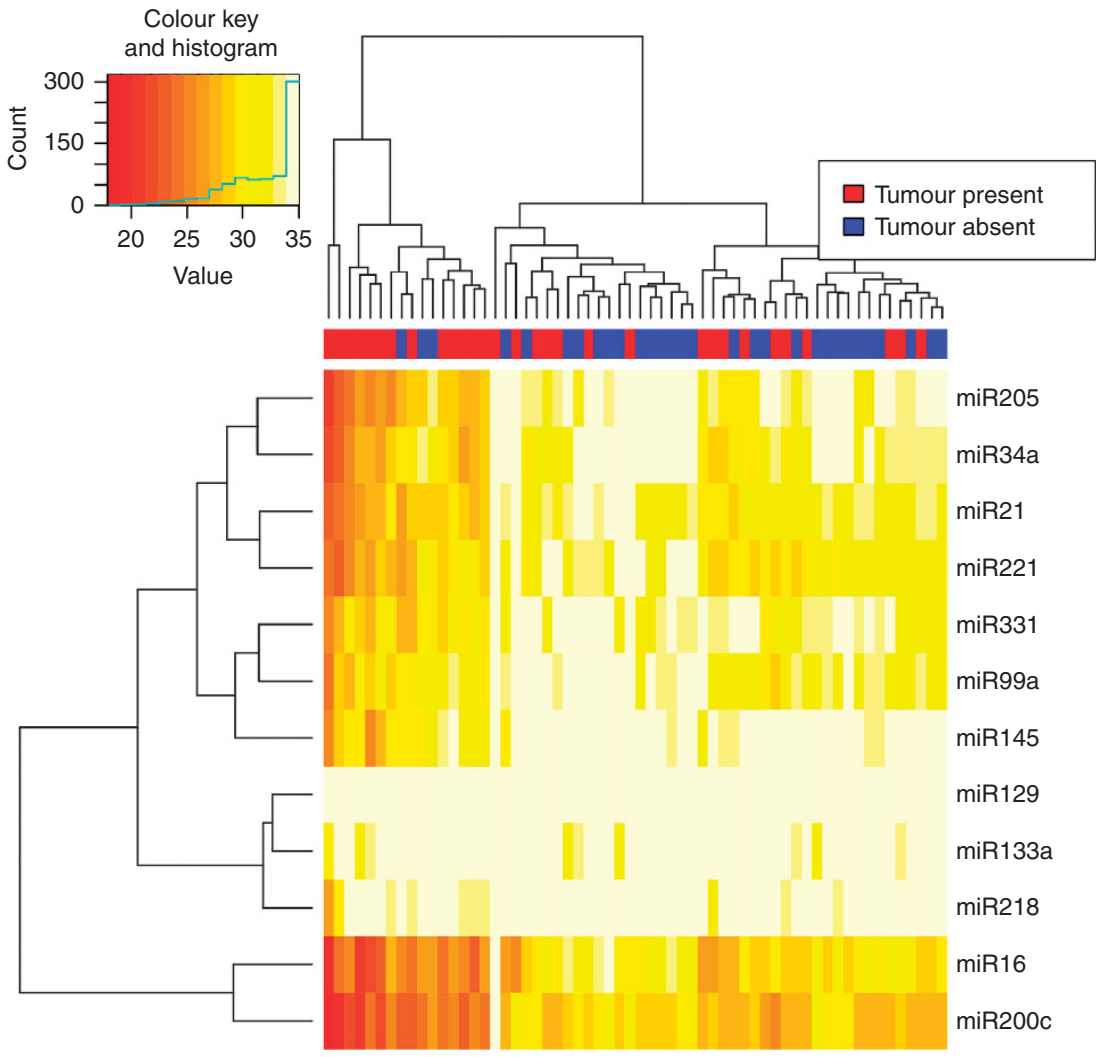

Figure 1. Heatmap of miRNA expression in urine ( $\mathrm{C} t$, denoted as value) for patients with active UCB (tumour present) and patients with a previous history of UCB that have not recurred (tumour absent). 
distinguishing samples with tumour present $v$ s those with tumour absent, we applied a three-fold classification scheme, repeated 100 times to measure those miRNAs which were most important for each iteration. Supplementary Table S4 provides a summary of the number of times each miRNA was used in these classifiers. From this, we were able to derive that miR16, miR200c, miR205, miR21, miR221 and miR34a provided the ability to predict the presence of a tumour with an $\mathrm{AUC}=0.85$ (Figure 2B).

Next, we validated these six miRNAs in an independent cohort of bladder cancer patients to check the reproducibility of this performance. The clinical and pathological characteristics of the validation cohort are shown in Supplementary Table S5. Both the groups were age and sex matched. The majority (52\%) of tumours were Ta tumours with $40 \%$ and $60 \%$ low-grade and high-grade
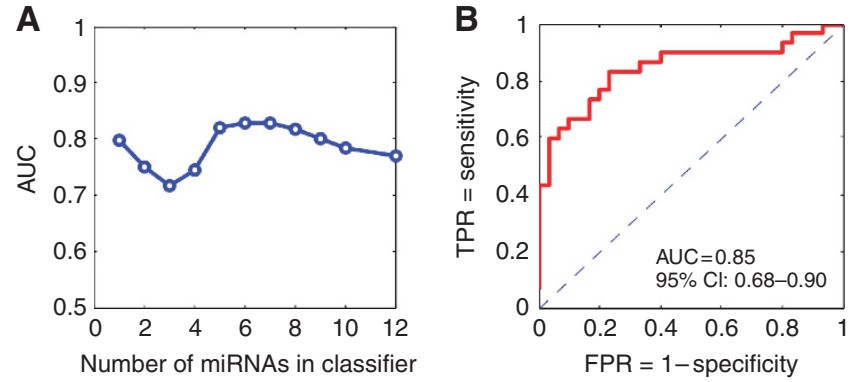

Figure 2. Feature selection and performance of miRNAs as biomarkers for predicting the presence of UCB in the discovery data set. (A) This figure displays the area under the ROC curve ( $y$ axis) of classifiers using different number of miRNAs during prediction ( $x$ axis). (B) ROC curve using the six miRNA panels to distinguish tumour present $v s$ benign tissues. tumours, respectively. No patients who had a negative cystoscopy had tumour recurrence within 12 months.

The miRNAs were detected in a high proportion of samples with miR 16, 21 and 200c detected in all samples in both cohorts. All miRNAs showed higher expression in the recurrence group as represented by lower logarithmic amplification thresholds (Ct) (Figure 3). We applied our classifier for predicting bladder cancer recurrence trained on the discovery cohort to the validation cohort resulting in an $\mathrm{AUC}=0.74$, showing that our panel of six miRNAs provide a robust and reproducible biomarker (Figure 4). In addition, we also tested the ability of the miRNAs to predict the presence of tumours for different subsets of our samples to deduce the effect of tumour size, presentation, $\mathrm{T}$ stage and grade on performance of the classifier. The best performance was observed when attempting to detect $\mathrm{T} 1$-stage tumours $(\mathrm{AUC}=0.92)$ (Figure 5). The most difficult tumours to detect were those with low-volume $(\mathrm{AUC}=0.69)$ disease. For prediction of recurrence in a clinical setting the most appropriate operational point on the ROC curve from the validation cohort is at sensitivity $88 \%$ and specificity $48 \%$. This results in a negative predicted value of $75 \%$ and positive predicted value of $63 \%$. This implies that if all patients who would have been correctly predicted as not having cancer recurrence, could have been spared a cystoscopy, cystoscopy rates in the independent validation cohort would have been reduced by $30 \%$. Of all significant tumours (large, invasive or high grade), only two high-grade cancers would have been missed.

In order to determine the biological relevance of these miRNAs we conducted a complementary study using RNA-seq-based UCB gene expression profiling, miRNA target prediction analysis, and database mining. Changes in gene expression between normal bladder epithelia and UCB were identified by RNA-seq analysis of an independent cohort of 21 patients (clinical characteristics
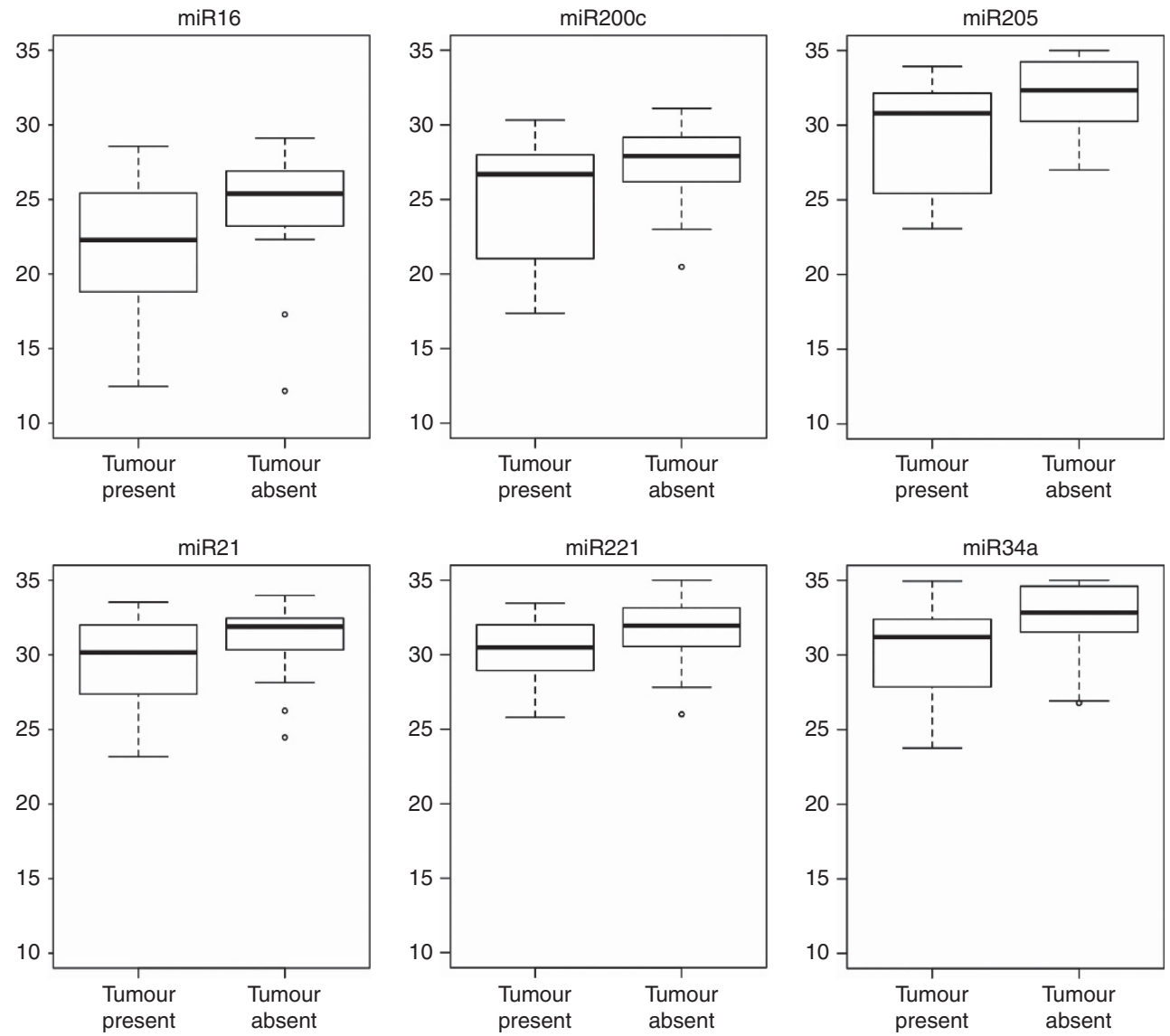

Figure 3. Box plots representing the expression ( $y$ axis, $\mathrm{Ct}$ ) of the six miRNAs in urine of patients from the validation cohort. Lower $\mathrm{Ct}$ depicts higher expression. 
summarised in Supplementary Table S6). Using the six miRNAs as part of our biomarker panel, we used a suite of miRNA target prediction tools to determine the set of genes targeted by the six miRNAs, to derive a predicted set of 82 candidate genes predicted to be regulated by the six miRNAs. Given that these miRNAs were all upregulated in samples, which had UCB present, we would expect that the majority of the targets of these miRNAs would have downregulated expression in tumour tissues, relative to benign tissues. To test this, we observed the expression of these target genes in our gene expression study, as well as three other bladder

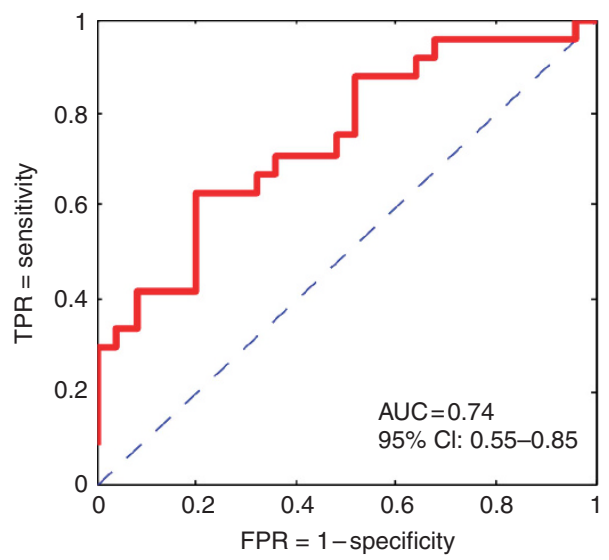

Figure 4. ROC curve of six miRNA classifiers applied to samples from the validation cohort. cancer expression studies (Dyrskjøt et al, 2004; Sanchez-Carbayo et al, 2006; Lee et al, 2010) using data downloaded from Oncomine (Rhodes et al, 2007). These studies provide comparative gene expression information for an additional 311 UCB samples (superficial and infiltrating) against 81 normal epithelial controls. Unsupervised hierarchical cluster analysis of our 82 candidate genes co-coordinately regulated miRNA targets revealed that the vast majority exhibit a significant reduction in both superficial and infiltrating UCB across the three studies (Figure 6). We ranked the degree of gene dysregulation across all studies by average percentile to identify those candidates that were most likely to contribute to UCB initiation and/or progression. Within the top 11 targets, we found five that have been shown to exhibit a tumour suppressor role in various cancers including RECK (Hirata et al, 2012), DMD (Schmidt et al, 2011), FOXF1 (Lo et al, 2010), ITIH5 (Veeck et al, 2008) and PTCH1 (Li et al, 2012). Interestingly, when the miRNA regulatory candidates were ranked according to our expression studies there are 6 genes in the top 25 that do not have expression data recorded in Oncomine and 3 of these also have potential tumour suppressor roles including PI16, (Crawford et al, 2008), AHRR (Zudaire et $a l, 2008$ ) and FAT3 (Katoh, 2012).

\section{DISCUSSION}

A lack of urinary biomarkers and reliance on cystoscopy for detection of bladder cancer underpin the high cost and morbidity of bladder cancer detection and surveillance. In this study, we have shown that profiling for miRNAs in urine can yield a signature that
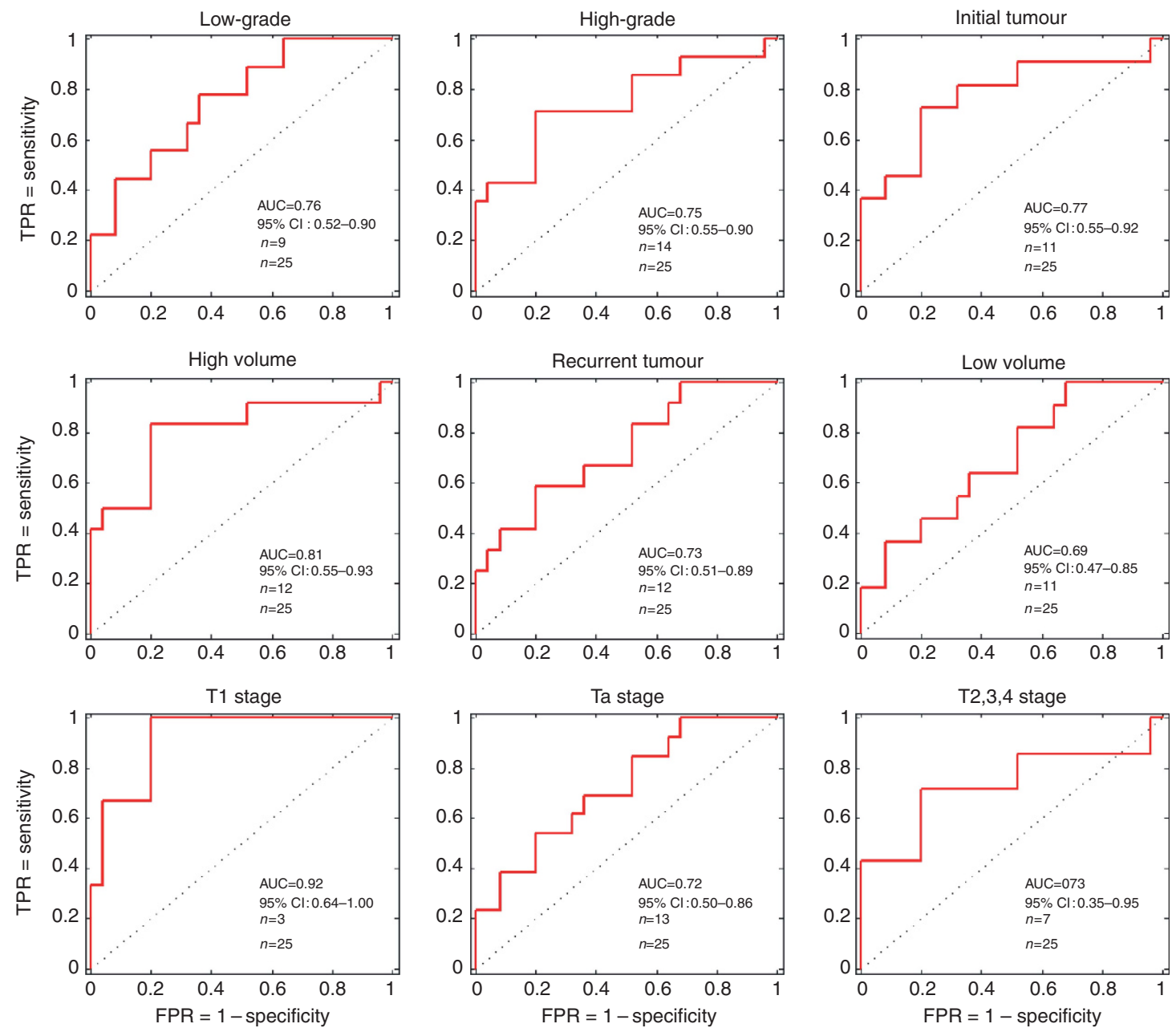

Figure 5. ROC curves for prediction using the six miRNA panels for different subsets of samples split by tumour characteristics. 


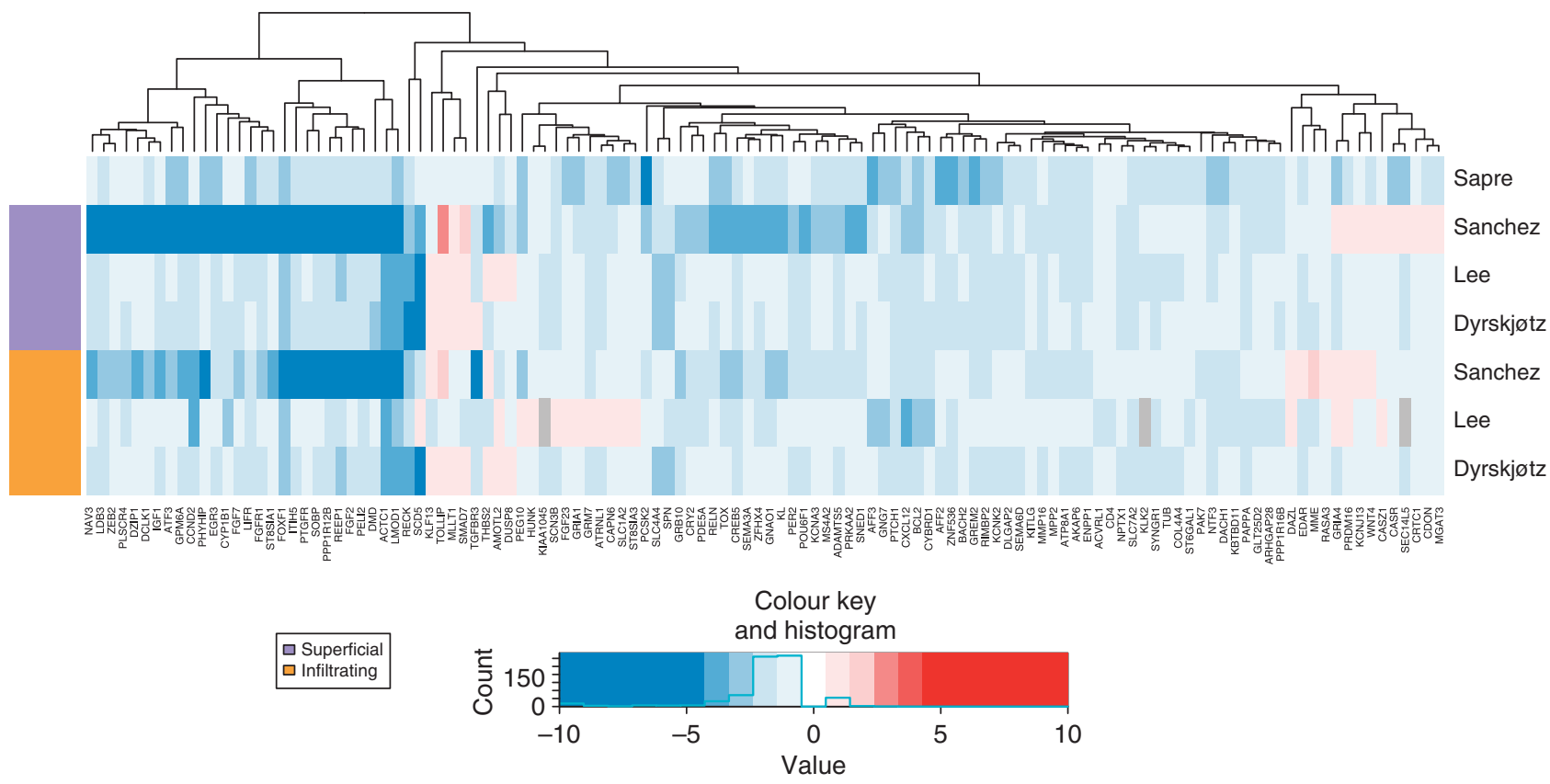

Figure 6. Heatmap of predicted miRNA target genes. The heatmap represents fold changes for predicted miRNA target genes in superficial vs infiltrating expression sets. Our RNA-seq data set (Sapre; unpublished) is shown compared with data sets obtained from Oncomine (Dyrskjøt et al, 2004; Sanchez-Carbayo et al, 2006; Lee et al, 2010). Genes were clustered using hierarchical clustering using Euclidian distance and complete agglomeration.

shows promising performance for non-invasive detection of bladder cancer especially in the surveillance setting. When comparing patients with active bladder cancer to non-recurrers, a 6-miRNA signature (miR 16, 21, 34a, 200c, 205, 221) was able to predict presence of active cancer with impressive performance $(\mathrm{AUC}=0.85)$ and this validated successfully in an independent cohort $(A U C=0.74)$. The performance of this miRNA classifier was significantly better for larger tumours $(\mathrm{AUC}=0.81$ vs $\mathrm{AUC}=0.69)$, and in tumours with higher T-stage $(\mathrm{AUC}=0.92$ vs $\mathrm{AUC}=0.72$ ).

Non-invasive blood and urine-based biomarkers are being investigated in several cancers. However, as the majority of bladder cancers are superficial, blood-based markers are less likely to be useful to detect tumours. In addition, since bladder tumours are in direct contact with urine, it represents the ideal body fluid for profiling of non-invasive biomarkers of bladder cancer. Gene-based biomarkers are likely to show significantly better performance in this regard as thousands of genetic changes can be accurately detected at once compared with lower throughput protein-based biomarkers. Protein-based biomarkers are also more likely to be affected by benign conditions such as infection and inflammation, which are very common in the lower urinary tract.

Other studies profiling miRNA expression in urine of bladder cancer patients have been performed, however, they have shown mixed results regarding the utility of miRNAs as urinary biomarkers of cancer. While these studies compared bladder cancer with noncancer control populations and found that miRNA can sensitively distinguish the bladder cancer samples from controls, this comparison is not a clinically relevant setting for predicting tumour recurrence (Hanke et al, 2010; Miah et al, 2012; Wang et al, 2012; Yun et al, 2012). Rather, a comparison of urine from patients with a recurrence $v s$ those who have had a previous history of bladder cancer but are disease free at time of collection, is a more suitable cohort. In addition, the specificity in several studies has been low and this may be due to heterogeneous control groups, which include patients with haematuria, other benign urological conditions, benign inflammatory and infective changes.
To our knowledge, few studies have assessed biomarkers for bladder recurrence using a suitable patient cohort. Yun et al (2012), provided the first indication that miRNAs could be used in a surveillance setting, showing that miR-200a provided prognostic potential in predicting recurrence, however, these findings were not validated in an independent cohort. Su et al (2014) reported the first attempt to predict bladder cancer recurrence using methylation profiling of three target genes in urine from a clinically relevant surveillance patient cohort. In this important study, they analysed 368 urine sediment samples serially collected from 90 patients for the methylation status of the three targeted regions and found an impressive discrimination between patients with and without cancer with AUCs of 0.90 and 0.95 , sensitivities ranging from 80 to $86 \%$ and specificities from 89 to $97 \%$ from their testing and validation sets, respectively. However, as the validation set was derived from the same patient cohort from which, the classifier was generated, there remains the possibility that the performance of the model may be artificially inflated due to over-fitting, a phenomenon that can only be avoided with a truly independent validation group.

We attempted to address these problems by using balanced cohorts of recurrers to non-recurrers, as well as using an independent validation cohort. The importance of independent validation of gene expression signatures has been highlighted previously (Lauss et al, 2010) and remains one of the central reasons that despite many promising investigational biomarkers, none has been translated to the clinic in the management of bladder cancer (Hong et al, 2014; Sapre et al, 2014). We also employed a principled approach for selection of miRNAs for profiling. As is seen in Supplementary Table S1, the miRNAs selected for profiling in this study systematically represent a diverse range of processes that contribute to tumourigenesis such as differentiation, apoptosis, cell cycle control, invasion and metastasis. Selected miRNAs were also expressed in several other epithelial cancers, with 10 having previous studies reporting involvement in bladder carcinogenesis and hence is likely to be more biologically relevant. Bladder cancer is a heterogeneous 
disease especially at different spectra of the disease and such a multi-marker strategy representing various pathways and processes is more likely to yield suitable biomarkers for bladder cancer (Bartsch et al, 2010; Mitra et al, 2013).

We have also used some novel ways of normalising data in this study. Initial use of RNU48 as an endogeneous control showed that the expression of RNU 48 was more varied amongst samples than the cancer miRNAs (data not shown). One of the reasons, RNU48 was unable to be used as an endogeneous control as in previous tissue studies could be due to the fact that the amount of RNA isolated from urine is very small compared with mRNA expression or the amount of miRNA isolated from tissue studies. Hence the novel approach of normalising to osmolality was chosen as urine osmolalities are significantly different based on clinical circumstance and can make large differences when measuring small amounts of biomarkers such as miRNAs in urine. Batch correction using prinicipal components analysis was used to correct for systematic errors in sample processing and analysis.

By employing these procedures and ensuring a high sensitivity (88\%) for detection of recurrers, albeit at the expense of a lessspecific test (48\%), we believe we provide a robust test that shows promise for use in a clinical setting. In addition, we also provide evidence for the functional implications of these miRNAs in disease recurrence. There is a growing body of evidence which indicates that a particular gene will be co-ordinately regulated by multiple miRNAs (Krek et al, 2005). Therefore we used gene expression profiles in a cohort of bladder cancer patients, to link our miRNA biomarkers, with their putative target genes. In order to select targets that were functionally relevant to UCB we only screened those genes that were downregulated in our expression analysis and then ranked them according to three other global UCB gene expression studies. Within the top $10 \%$ of ranked targets, nearly half have been shown to exhibit tumour suppressor function. Regardless of whether these are bona fide miR targets, the fact that they are both downregulated and operate in disparate pathways is supportive of a multifactorial etiology for UCB. Broader examination of the target list revealed five highly disregulated genes that are involved in hedgehog $(\mathrm{HH})$ signalling, namely PCTH1, FOXL1, BCL2, CCND2 and ZEB2. PCTH1 acts as the canonical HH receptor and FOXF1, BCL2, CCND2 and ZEB2 are targets of GLI1 transcription factor that mediates $\mathrm{HH}$ signalling (Katoh, 2009). It has been shown that the $\mathrm{HH}$ signalling pathway is constitutively activated in many urothelial cell lines and correlates with UCB progression (Fei et al, 2012). From this it would be expected that the downstream elements FOXL1, BCL2 CCND2 and ZEB2 should be upregulated. The fact that there are downregulated, however, is supportive of posttranscriptional regulation, perhaps by a mechanism that involves miRNA. In further considering the role of FOXF1, this gene also functions as an inhibitor of DNA replication, a process that increases the incidence of chromosomal aberrations including DNA rearrangements. Genomic instability is an early hallmark of UCB that distinguishes $\mathrm{Ta}$ from $\geqslant \mathrm{T} 1$ tumours. In addition to deregulation of a number of tumour suppressor genes we also observed repression of a number of oncogenic genes, particularly in the BCL2 and FGF (FGF2, FGF23, FGFR1, FGF7) pathways. While we feel that this is not likely to influence progression of UCB it is informative about the type of therapies that are unlikely to be effective for UCB treatment or management.

\section{CONCLUSION}

This study reveals the merits of screening for specific combinations of miRNAs in urine from bladder cancer patients in the surveillance setting as a potential tool for reducing the morbidity and costs associated with current procedures, and now merits prospective evaluation. Limitations of this study include a small amount of samples taken from patients with initial presentation and the limited number of samples used in the cohorts. A larger prospective study in the surveillance cohort is currently planned.

As far as we are aware this is also the first study to validate findings in an independent surveillance patient cohort and correlate it to gene expression in data from independent groups. This suggests that future validation studies with this panel of miRNAs as well potentially in combination with other urinary biomarkers (Su et al, 2014) may hold real promise for clinical translation in the not too distant future.

\section{CONFLICT OF INTEREST}

The authors declare no conflict of interest.

\section{REFERENCES}

Adam L, Zhong M, Choi W, Qi W, Nicoloso M, Arora A, Calin G, Wang H, Siefker-Radtke A, Mcconkey D, Bar-Eli M, Dinney C (2009) miR-200 expression regulates epithelial-to-mesenchymal transition in bladder cancer cells and reverses resistance to epidermal growth factor receptor therapy. Clin Cancer Res 15: 5060-5072.

Avritscher EB, Cooksley CD, Grossman HB, Sabichi AL, Hamblin L, Dinney CP, Elting LS (2006) Clinical model of lifetime cost of treating bladder cancer and associated complications. Urology 68: 549-553.

Bartsch G, Mitra AP, Cote RJ (2010) Expression profiling for bladder cancer: strategies to uncover prognostic factors. Expert Rev Anticancer Ther 10: 1945-1954.

Benjamini YAH (1995) Controlling the false discovery rate: a practical and powerful approach to multiple testing. J Royal Stat Soc 57: 289-300.

Botteman MF, Pashos CL, Redaelli A, Laskin B, Hauser R (2003) The health economics of bladder cancer: a comprehensive review of the published literature. Pharmacoeconomics 21: 1315-1330.

Burk U, Schubert J, Wellner U, Schmalhofer O, Vincan E, Spaderna S, Brabletz T (2008) A reciprocal repression between ZEB1 and members of the miR-200 family promotes EMT and invasion in cancer cells. EMBO Rep 9: 582-589.

Catto JW, Alcaraz A, Bjartell AS, De Vere White R, Evans CP, Fussel S, Hamdy FC, Kallioniemi O, Mengual L, Schlomm T, Visakorpi T (2011) MicroRNA in prostate, bladder, and kidney cancer: a systematic review. Eur Urol 59: 671-681.

Crawford NP, Walker RC, Lukes L, Officewala JS, Williams RW, Hunter KW (2008) The Diasporin pathway: a tumour progression-related transcriptional network that predicts breast cancer survival. Clin Exp Metastasis 25: 357-369.

Daniltchenko DI, Riedl CR, Sachs MD, Koenig F, Daha KL, Pflueger H, Loening SA, Schnorr D (2005) Long-term benefit of 5-aminolevulinic acid fluorescence assisted transurethral resection of superficial bladder cancer: 5-year results of a prospective randomized study. J Urol 174: 2129-2133; discussion 2133.

Dyrskjøt L, Kruhoffer M, Thykjaer T, Marcussen N, Jensen JL, Moller K, Orntoft TF (2004) Gene expression in the urinary bladder: a common carcinoma in situ gene expression signature exists disregarding histopathological classification. Cancer Res 64: 4040-4048.

Epstein JI, Amin MB, Reuter VR, Mostofi FK (1998) The World Health Organization/International Society of Urological Pathology consensus classification of urothelial (transitional cell) neoplasms of the urinary bladder. Bladder Consensus Conference Committee. Am J Surg Pathol 22: 1435-1448.

Fei DL, Sanchez-Mejias A, Wang Z, Flaveny C, Long J, Singh S, RodriguezBlanco J, Tokhunts R, Giambelli C, Briegel KJ, Schulz WA, Gandolfi AJ, Karagas M, Zimmers TA, Jorda M, Bejarano P, Capobianco AJ, Robbins DJ (2012) Hedgehog signaling regulates bladder cancer growth and tumourigenicity. Cancer Res 72: 4449-4458.

Garzon R, Calin GA, Croce CM (2009) MicroRNAs in Cancer. Annu Rev Med 60: $167-179$. 
Hanke M, Hoefig K, Merz H, Feller AC, Kausch I, Jocham D, Warnecke JM, Sczakiel G (2010) A robust methodology to study urine microRNA as tumour marker: microRNA-126 and microRNA-182 are related to urinary bladder cancer. Urol Oncol 28: 655-661.

Hirata H, Ueno K, Shahryari V, Tanaka Y, Tabatabai ZL, Hinoda Y, Dahiya R (2012) Oncogenic miRNA-182-5p targets Smad4 and RECK in human bladder cancer. PLoS One 7: e51056.

Hong MK, Sapre N, Phal PM, Macintyre G, Chin X, Pedersen JS, Ryan A, Kerger M, Costello AJ, Corcoran NM, Hovens CM (2014) Percutaneous image-guided biopsy of prostate cancer metastases yields samples suitable for genomics and personalised oncology. Clin Exp Metastasis 31: 159-167.

Jansson MD, Lund AH (2012) MicroRNA and cancer. Mol Oncol 6: 590-610.

Katoh M (2009) Transcriptional mechanisms of WNT5A based on NFkappaB, Hedgehog, TGFbeta, and Notch signaling cascades. Int J Mol Med 23: 763-769.

Katoh M (2012) Function and cancer genomics of FAT family genes (review). Int J Oncol 41: 1913-1918.

Khadra MH, Pickard RS, Charlton M, Powell PH, Neal DE (2000) A prospective analysis of 1,930 patients with hematuria to evaluate current diagnostic practice. J Urol 163: 524-527.

Kim D, Pertea G, Trapnell C, Pimentel H, Kelley R, Salzberg SL (2013) TopHat2: accurate alignment of transcriptomes in the presence of insertions, deletions and gene fusions. Genome Biol 14: R36.

Krek A, Grun D, Poy MN, Wolf R, Rosenberg L, Epstein EJ, Macmenamin P, Da Piedade I, Gunsalus KC, Stoffel M, Rajewsky N (2005) Combinatorial microRNA target predictions. Nat Genet 37: 495-500.

Kupfer P, Guthke R, Pohlers D, Huber R, Koczan D, Kinne RW (2012) Batch correction of microarray data substantially improves the identification of genes differentially expressed in rheumatoid arthritis and osteoarthritis. BMC Med Genomics 5: 23.

Langmead B, Trapnell C, Pop M, Salzberg SL (2009) Ultrafast and memoryefficient alignment of short DNA sequences to the human genome. Genome Biol 10: R25.

Lauss M, Ringner M, Hoglund M (2010) Prediction of stage, grade, and survival in bladder cancer using genome-wide expression data: a validation study. Clin Cancer Res 16: 4421-4433.

Lee JS, Leem SH, Lee SY, Kim SC, Park ES, Kim SB, Kim SK, Kim YJ, Kim WJ, Chu IS (2010) Expression signature of E2F1 and its associated genes predict superficial to invasive progression of bladder tumours. J Clin Oncol 28: 2660-2667.

Li Y, Zhang D, Chen C, Ruan Z, Huang Y (2012) MicroRNA-212 displays tumour-promoting properties in non-small cell lung cancer cells and targets the hedgehog pathway receptor PTCH1. Mol Biol Cell 23: 1423-1434.

Lo PK, Lee JS, Liang X, Han L, Mori T, Fackler MJ, Sadik H, Argani P, Pandita TK, Sukumar S (2010) Epigenetic inactivation of the potential tumour suppressor gene FOXF1 in breast cancer. Cancer Res 70: 6047-6058.

Lohse M, Bolger AM, Nagel A, Fernie AR, Lunn JE, Stitt M, Usadel B (2012) RobiNA: a user-friendly, integrated software solution for RNA-Seq-based transcriptomics. Nucleic Acids Res 40: W622-W627.

Miah S, Dudziec E, Drayton RM, Zlotta AR, Morgan SL, Rosario DJ, Hamdy FC, Catto JW (2012) An evaluation of urinary microRNA reveals a high sensitivity for bladder cancer. Br J Cancer 107: 123-128.

Mitra AP, Castelao JE, Hawes D, Tsao-Wei DD, Jiang X, Shi SR, Datar RH, Skinner EC, Stein JP, Groshen S, Yu MC, Ross RK, Skinner DG, Cortessis VK, Cote RJ (2013) Combination of molecular alterations and smoking intensity predicts bladder cancer outcome: a report from the Los Angeles Cancer Surveillance Program. Cancer 119: 756-765.
Mongroo PS, Rustgi AK (2010) The role of the miR-200 family in epithelialmesenchymal transition. Cancer Biol Ther 10: 219-222.

Quinlan AR, Hall IM (2010) BEDTools: a flexible suite of utilities for comparing genomic features. Bioinformatics 26: 841-842.

Rhodes DR, Kalyana-Sundaram S, Mahavisno V, Varambally R, Yu J, Briggs BB, Barrette TR, Anstet MJ, Kincead-Beal C, Kulkarni P, Varambally S, Ghosh D, Chinnaiyan AM (2007) Oncomine 3.0: genes, pathways, and networks in a collection of 18,000 cancer gene expression profiles. Neoplasia 9: 166-180.

Robinson MD, Mccarthy DJ, Smyth GK (2010) edgeR: a Bioconductor package for differential expression analysis of digital gene expression data. Bioinformatics 26: 139-140.

Sanchez-Carbayo M, Socci ND, Lozano J, Saint F, Cordon-Cardo C (2006) Defining molecular profiles of poor outcome in patients with invasive bladder cancer using oligonucleotide microarrays. J Clin Oncol 24: 778-789.

Sangar VK, Ragavan N, Matanhelia SS, Watson MW, Blades RA (2005) The economic consequences of prostate and bladder cancer in the UK. BJU Int 95: 59-63.

Sapre N, Anderson PD, Costello AJ, Hovens CM, Corcoran NM (2014) Gene-based urinary biomarkers for bladder cancer: an unfulfilled promise? Urol Oncol 32: 48 e9-17.

Schmidt WM, Uddin MH, Dysek S, Moser-Thier K, Pirker C, Hoger H, Ambros IM, Ambros PF, Berger W, Bittner RE (2011) DNA damage, somatic aneuploidy, and malignant sarcoma susceptibility in muscular dystrophies. PLoS Genet 7: e1002042.

Schrag D, Hsieh LJ, Rabbani F, Bach PB, Herr H, Begg CB (2003) Adherence to surveillance among patients with superficial bladder cancer. J Natl Cancer Inst 95: 588-597.

Su SF, De Castro Abreu AL, Chihara Y, Tsai Y, Andreu-Vieyra C, Daneshmand S, Skinner EC, Jones PA, Siegmund KD, Liang G (2014) A panel of three markers hyper- and hypomethylated in urine sediments accurately predicts bladder cancer recurrence. Clin Cancer Res 20: 1978-1989.

Sylvester RJ, Van Der Meijden AP, Oosterlinck W, Witjes JA, Bouffioux C, Denis L, Newling DW, Kurth K (2006) Predicting recurrence and progression in individual patients with stage Ta T1 bladder cancer using EORTC risk tables: a combined analysis of 2596 patients from seven EORTC trials. Eur Urol 49: 466-475.

Veeck J, Chorovicer M, Naami A, Breuer E, Zafrakas M, Bektas N, Durst M, Kristiansen G, Wild PJ, Hartmann A, Knuechel R, Dahl E (2008) The extracellular matrix protein ITIH5 is a novel prognostic marker in invasive node-negative breast cancer and its aberrant expression is caused by promoter hypermethylation. Oncogene 27: 865-876.

Wang G, Chan ES, Kwan BC, Li PK, Yip SK, Szeto CC, Ng CF (2012) Expression of microRNAs in the urine of patients with bladder cancer. Clin Genitourin Cancer 10: 106-113.

Yun SJ, Jeong P, Kim WT, Kim TH, Lee YS, Song PH, Choi YH, Kim IY, Moon SK, Kim WJ (2012) Cell-free microRNAs in urine as diagnostic and prognostic biomarkers of bladder cancer. Int J Oncol 41: 1871-1878.

Zudaire E, Cuesta N, Murty V, Woodson K, Adams L, Gonzalez N, Martinez A, Narayan G, Kirsch I, Franklin W, Hirsch F, Birrer M, Cuttitta F (2008) The aryl hydrocarbon receptor repressor is a putative tumour suppressor gene in multiple human cancers. J Clin Invest 118: 640-650.

This work is published under the standard license to publish agreement. After 12 months the work will become freely available and the license terms will switch to a Creative Commons AttributionNonCommercial-Share Alike 4.0 Unported License.

Supplementary Information accompanies this paper on British Journal of Cancer website (http://www.nature.com/bjc) 